\title{
A tower condition characterizing normality
}

\author{
Lars KADISON
}

(Received January 30, 2014; Revised March 19, 2014)

\begin{abstract}
We define left relative $\mathrm{H}$-separable tower of rings and continue a study of these begun by Sugano. It is proven that a progenerator extension has right depth 2 if and only if the ring extension together with its right endomorphism ring is a left relative $\mathrm{H}$-separable tower. In particular, this applies to twisted or ordinary Frobenius extensions with surjective Frobenius homomorphism. For example, normality for Hopf subalgebras of finite-dimensional Hopf algebras is also characterized in terms of this tower condition.
\end{abstract}

Key words: Frobenius extension, H-separable extension, normal subring, induced characters, subring depth, Hopf subalgebra.

\section{Introduction and Preliminaries}

Depth two is a bimodule condition on subrings that is equivalent to usual notions of normality for subgroups [4], Hopf subalgebras [2] and semisimple complex subalgebra pairs [6]; in addition, depth two is a condition of normality for a ring extension that makes it a Galois extension with respect to a right bialgebroid coaction [16], [18]. The right depth two condition on an algebra extension $A \supseteq B$ is that the natural $A$ - $B$-bimodule $A \otimes_{B} A$ is isomorphic to a direct summand of a natural $A$-B-bimodule $A \oplus \cdots \oplus A$ : in symbols this is ${ }_{A} A \otimes_{B} A_{B} \oplus * \cong{ }_{A} A_{B}^{n}$. If $A$ is a finite-dimensional Hopf algebra and $B$ is a Hopf subalgebra of $A$, it is shown in [2] that $A \supseteq B$ has right (or left) depth 2 if and only if $B$ is a normal Hopf subalgebra of $A$ (i.e., $B$ is invariant under either the left or right adjoint actions). If $A$ is a finite-dimensional group algebra $\mathbb{C} G$, its module theory is determined by the character theory of $G$, and the right depth two condition on a group subalgebra $B=\mathbb{C} H$ in $A$ is determined by a matrix inequality condition on the induction-restriction table for the irreducible characters of $G$ and subgroup $H$; for more on this, depth greater than 2 as well as modular representations, see [5], [6], [4], [7], [8], [9].

For the reasons just given we call a subring $B \subseteq A$ satisfying the right depth 2 condition above a right normal subring (and the ring extension 
$A \supseteq B$ a right normal extension), the details appearing in Definition 1.7. We find a characterization of normal Frobenius extensions with surjective Frobenius homomorphism (such as finite Hopf-Galois extensions with surjective trace map [15]), in terms of an old one-sided H-separability condition on a tower of rings $A \supseteq B \supseteq C$ appearing in Sugano's [25, Lemma 1.2]. This condition is interesting and we gather into "Sugano's Theorem" (Theorem 1.8) the results for a tower satisfying this condition in [25], providing a different proof. We show in Section 3 that a ring extension $A \supseteq B$ with the natural module $A_{B}$ a progenerator, together with its right endomorphism ring End $A_{B}$, forms a tower satisfying Sugano's condition, called "left relative H-separable," if and only if $A \supseteq B$ is a right normal extension. In Corollary 3.5 it is noted that Theorem 3.2 establishes the same tower characterization of normality for $\beta$-Frobenius extensions with surjective Frobenius homomorphism. For example, an arbitrary Hopf subalgebra of a finite-dimensional Hopf algebra is such a twisted Frobenius extension: then Corollary 3.6 characterizes a normal Hopf subalgebra in terms of its right endomorphism algebra. A new proof that right normality is equivalent to left normality for Frobenius extensions with the surjectivity condition is noted in Corollary 3.4.

\subsection{H-separable extensions}

A ring extension $A \supseteq B$ is H-separable if $A \otimes_{B} A \oplus * \cong A^{n}$ as natural $A$-bimodules [11]. The notion of $\mathrm{H}$-separability extends certain nice results for Azumaya algebras to ring extensions. For example, the Azumaya isomorphism of the enveloping algebra and the endomorphism algebra is extended for an $\mathrm{H}$-separable ring extension $A \supseteq B$ to a bimodule isomorphism, $A \otimes_{B} A \cong \operatorname{Hom}\left(R_{Z}, A_{Z}\right)$ where $R$ is the centralizer of $B$ in $A$ and $Z$ is the center of $A$ [10]. One also shows that $A \supseteq B$ is a separable extension, and if this is additionally split, that $R$ is a separable algebra over $Z$ [10], [25], [14]. Any bimodule $M$ over $A$ has a generalized Azumaya isomorphism $M^{A} \otimes_{Z} R \cong M^{B}$ between the $A$ - and $B$-centralized elements of $M$.

$\mathrm{H}$-separable extension theory was one of the motivational models for [16] which extends to ring theory the notion of depth 2 for free Frobenius extensions in [15] (see [16, Examples 3.6, 4.8, 5.8], another toy model being Lu's Hopf algebroids on an algebra). Examples of $\mathrm{H}$-separable extensions come from tensoring Azumaya algebras with other algebras, or looking at certain subalgebras within Azumaya algebras; certainly group algebra and 
Hopf algebra extensions are trivial if H-separable, which is true generally [22] but easier to prove with characters (see Proposition 2.6 in this paper). The more general notion of depth two ring extension welcomes examples from Hopf-Galois extensions including normal Hopf subalgebras; indeed, depth two ring extension is equivalent, with one other condition (balanced module), to a Galois extension with bialgebroid coactions [16], [18], where bialgebroid is the good generalization of bialgebra from algebras to ring extensions.

An H-separable extension $A \supseteq B$ is the H-depth $n=1$ case of odd minimal H-depth $d_{H}(B, A)=2 n-1$ where $A^{\otimes_{B} n} \sim A^{\otimes_{B}(n+1)}$ as $A$-bimodules ([20], see below in this section for H-equivalent modules). We show in Propositon 2.2 that a relative separable and $\mathrm{H}$-separable tower $A \supseteq B \supseteq C$ has equality of minimal H-depth, $d_{H}(B, A)=d_{H}(C, A)$. We note a different proof of Sugano's theorem 1.8 (cf. [25]) that shows that in such a relative $\mathrm{H}$-separable tower there is a close relation between depth 1 (centrally projective) and H-depth 1 (H-separable) extensions $B \supseteq C$ and $A^{C} \supseteq A^{B}$, as well as split and separable extensions.

The following unpublished characterization of H-separable extensions is useful below.

Proposition 1.1 Let $A \mid B$ be a ring extension. Then $A \mid B$ is $H$-separable if and only if for each $A$-module $N$, its restriction and induction satisfies Ind $_{B}^{A} \operatorname{Res}_{B}^{A} N \oplus * \cong N^{m}$ for some $m \in \mathbb{N}$ via two natural transformations. Consequently, if $A \mid B$ is H-separable and $A$-modules $V, W$ satisfy $V_{B} \oplus * \cong$ $W_{B}$, then $V_{A} \oplus * \cong W_{A}^{m}$ for some $m \in \mathbb{N}$.

Proof. The second statement follows from the fact that $A \mid B$ is also separable, so that $V \otimes_{B} A \rightarrow V, v \otimes a \mapsto v a$ is a natural split epi. Note that $V \otimes_{B} A \oplus * \cong W \otimes_{B} A$, so that the second statement follows from the characterization in the first statement.

$\left(\Rightarrow\right.$ ) Since $A \otimes_{B} A \oplus * \cong A^{n}$ as $A$-bimodules, the implication follows from tensoring this by $N \otimes_{A}-$. Naturality follows from looking more carefully at the mappings, starting with a module homomorphism $g: N_{A} \rightarrow$ $N_{A}^{\prime}$. Another characterization of H-separability is that there are elements $e_{i} \in\left(A \otimes_{B} A\right)^{A}$ and $r_{i} \in A^{B}(i=1, \ldots, n)$ such that $1 \otimes_{B} 1=\sum_{i} r_{i} e_{i}$. For each module $M_{A}$, define natural transformations $\tau_{M}: M \otimes_{B} A \rightarrow M_{A}^{n}$ by $\tau_{M}\left(m \otimes_{B} a\right)=\left(m r_{1} a, \ldots, m r_{n} a\right)$, and $\sigma_{M}: M_{A}{ }^{n} \rightarrow M \otimes_{B} A$ by $\sigma_{M}\left(m_{1}, \ldots, m_{n}\right)=\sum_{i} m_{i} e_{i}$; note that $\sigma_{M} \tau_{M}=\operatorname{id}_{M \otimes_{B} A}$ and the naturality 
commutative square follows readily.

$(\Leftarrow)$ Let $N=A$ in the hypothesis using natural transformations (as above) $\sigma_{N}$ and $\tau_{N}$. Then there are $A$-bimodule homomorphisms (from naturality) $\tau_{A}: A \otimes_{B} A \rightarrow A^{m}$ and $\sigma_{A}: A^{m} \rightarrow A \otimes_{B} A$ such that $\left.\sigma_{A}\left(\tau_{A}\left(1 \otimes_{B} 1\right)\right)\right)=1 \otimes_{B} 1$. Then $\tau_{A}\left(1 \otimes_{B} 1\right)=\left(r_{1}, \ldots, r_{m}\right) \in\left(A^{B}\right)^{m}$ and, denoting the canonical basis of $A^{m}$ by $\left\{c_{1}, \ldots, c_{m}\right\}, \sigma_{A}\left(c_{i}\right)=e_{i} \in\left(A \otimes_{B} A\right)^{A}$. Since $\tau_{A}$ is a section of $\sigma_{A}$, the equation $1 \otimes_{B} 1=\sum_{i=1}^{m} r_{i} e_{i}$ follows; thus $A \mid B$ is H-separable.

\subsection{Left relative separable ring towers}

In this paper a tower of rings $A \supseteq B \supseteq C$ is a unital associative ring $A$ with subring $B$, and $C$ a subring of $B$, so that $1_{C}=1_{B}=1_{A}$, which is denoted by 1 . Sugano in [25] defines $B$ to be a left relative separable extension of $C$ in $A$ (or briefly, a left relative separable tower) if the $B-A$ epimorphism $\mu: B \otimes_{C} A \rightarrow A$, defined by $\mu(b \otimes a)=b a$, is split; equivalently, there is a $B$-central element $e \in\left(B \otimes_{C} A\right)^{B}$ such that $e^{1} e^{2}=1$ (where $e=e^{1} \otimes e^{2}$ is modified Sweedler notation suppressing a finite sum of simple tensors). Similarly one defines $B$ to be a right relative separable extension of $C$ in $A$ by requiring $\mu: A \otimes_{C} B \rightarrow A$ to be a split $A$ - $B$-epimorphism. The next lemma notes that a separable extension $B \supseteq C$ always give rise to a left and right relative separable extension of $C$ in any over-ring $A$.

Lemma 1.2 Let $A \supseteq B \supseteq C$ be a tower of rings. If $B \supseteq C$ is a separable extension, then $B$ is a left and right relative separable extension of $C$ in $A$. Conversely, if $A \supseteq B$ is a split extension and $B$ is a left or right relative separable extension of $C$ in $A$, then $B \supseteq C$ is a separable extension.

Proof. Let $e \in\left(B \otimes_{C} B\right)^{B}$ satisfy $\mu(e)=e^{1} e^{2}=1$, the separability condition on $B \supseteq C$. Then $e \in\left(B \otimes_{C} A\right)^{B} \cap\left(A \otimes_{C} B\right)^{B}$ defines mappings $a \mapsto a e$ and $a \mapsto e a$ splitting $\mu_{r}: A \otimes_{C} B \rightarrow A$ and $\mu_{\ell}: B \otimes_{C} A \rightarrow A$, respectively.

Suppose $E:{ }_{B} A_{B} \rightarrow{ }_{B} B_{B}$ is a bimodule projection (equivalently, $E(1)=1$ ), and $\mu: B \otimes_{C} A \rightarrow A$ is a split $B$ - $A$-epimorphism (by $\left.\sigma: A \rightarrow B \otimes_{C} A\right)$. Then $e=\sigma(1)$ is in $\left(B \otimes_{C} A\right)^{B}$ satisfying $e^{1} e^{2}=1$. Note then that $e^{1} \otimes_{C} E\left(e^{2}\right)$ is a separability element for $B \supseteq C$. A similar argument for a right relative separable tower shows that $B \supseteq C$ is separable.

For example, suppose $G>H>J$ is a tower of finite groups (i.e., $H$ and $J$ are subgroups of $G$ where $J \subseteq H$ ). Since group algebra extensions 
are always split, the lemma implies that a tower of group algebras $K G \supseteq$ $K H \supseteq K J$ over a commutative ring $K$ is left or right relative separable if and only if $K H \supseteq K J$ is separable if and only if $|H: J| 1$ is an invertible element in $K$.

The lemma below provides nontrivial examples of relative separable towers; its proof is easy and therefore omitted.

Lemma 1.3 Suppose $A \supseteq C$ is a separable extension with separability element $e \in B \otimes_{C} A$ for some intermediate subring $B$ of $A$ containing $C$. Then $B$ is a left relative separable extension of $C$ in $A$.

Example 1.4 Let $K$ be a commutative ring and $A=M_{n}(K)$ the full $K$ algebra of $n \times n$ matrices. Let $e_{i j}$ denote the matrix units $(i, j=1, \ldots, n)$. Any of the $n$ elements $e_{j}=\sum_{i=1}^{n} e_{i j} \otimes_{K} e_{j i}$ are separability idempotents for $A$. This is also an example of a (symmetric) Frobenius algebra with trace map $T: A \rightarrow K$ having dual bases $e_{i j}, e_{j i}$.

Let $B_{1}, B_{2}$ denote the upper and lower triangular matrix algebras respectively (both of rank $n(n+1) / 2$ ). Then $B_{1}$ and $B_{2}$ are right, respectively left, relative separable algebras in $M_{n}(K)$, since $e_{1} \in A \otimes_{K} B_{1} \cap B_{2} \otimes_{K} A$.

\subsection{Left relative $\mathbf{H}$-separable ring towers}

In the same paper [25], Sugano considers a related condition on a tower of rings $A \supseteq B \supseteq C$, given by the condition on $B$ - $A$-bimodules,

$$
{ }_{B} B \otimes_{C} A_{A} \oplus * \cong{ }_{B} A_{A}^{n},
$$

for some $n \in \mathbb{N}$, i.e., $B \otimes_{C} A$ is isomorphic to a direct summand of $A \oplus \cdots \oplus A$ as natural $B$-A-bimodules. We define $B$ to be a left relative $H$-separable extension of $C$ in $A$ (or briefly refer to a left relative $H$-separable tower) if it satisfies the condition in (1). Note that if $A=B$ the condition in (1) is that of H-separability of $B \supseteq C$; if $B=C$, the condition becomes trivially satisfied by any ring extension $A \supseteq B$. We note a lemma similar to the one above.

Lemma 1.5 Let $A \supseteq B \supseteq C$ be a tower of rings. If $B \supseteq C$ is an $H$-separable extension, then $B$ is a left (and right) relative $H$-separable extension of $C$ in $A$.

Proof. Given the H-separability condition on the natural $B$ - $B$-bimodules, $B \otimes_{C} B \oplus * \cong B^{m}$, we tensor this from the right by the additive functor 
$-\otimes_{B} A_{A}$. After a cancellation of the type $B \otimes_{B} A \cong A$, the condition in (1) results. Tensoring similarly by additive functor $A \otimes_{B}$ - from the category of $B$ - $B$-bimodules into the category of $A$ - $B$-bimodules results in the obvious right relative $\mathrm{H}$-separable extension condition $\left({ }_{A} A \otimes_{C} B_{B} \oplus * \cong{ }_{A} A_{B}^{n}\right)$ on $B$ over $C$ in $A$.

Note that if $B$ is an H-separable extension of $C$, then $B$ is left and right relative separable and relative $\mathrm{H}$-separable extension of $C$ in any over-ring $A$, since $\mathrm{H}$-separable extensions are separable extensions [14] (and applying both lemmas).

\subsection{Preliminaries on subring normality and depth}

Let $A$ be a unital associative ring. The category of right modules over $A$ will be denoted by $\mathcal{M}_{A}$. Two modules $M_{A}$ and $N_{A}$ are $H$-equivalent (or similar) if $M \oplus * \cong N^{q}$ and $N \oplus * \cong M^{r}$ for some $r, q \in \mathbb{N}$ (sometimes briefly denoted by $M \sim N$ ). It is well-known that H-equivalent modules have Morita equivalent endomorphism rings.

Let $B$ be a subring of $A$ (always supposing $1_{B}=1_{A}$ ). Consider the natural bimodules ${ }_{A} A_{A},{ }_{B} A_{A},{ }_{A} A_{B}$ and ${ }_{B} A_{B}$ where the last is a restriction of the preceding, and so forth. Denote the tensor powers of ${ }_{B} A_{B}$ by $A^{\otimes_{B} n}=$ $A \otimes_{B} \cdots \otimes_{B} A$ for $n=1,2, \ldots$, which is also a natural bimodule over $B$ and $A$ in any one of four ways; set $A^{\otimes_{B} 0}=B$ which is only a natural $B$ - $B$ bimodule.

Definition 1.6 If $A^{\otimes_{B}(n+1)}$ is H-equivalent to $A^{\otimes_{B} n}$ as $X$ - $Y$-bimodules, one says $B \subseteq A$ (or $A \supseteq B$ ) has

- depth $2 n+1$ if $X=B=Y$;

- left depth $2 n$ if $X=B$ and $Y=A$;

- right depth $2 n$ if $X=A$ and $Y=B$;

- H-depth $2 n-1$ if $X=A=Y$.

(Valid for even depth and H-depth if $n \geq 1$ and for odd depth if $n \geq 0$.) Note that $B \subseteq A$ having depth $n$ implies it has depth $n+1$. Similarly if $B \subseteq A$ has H-depth $2 n-1$, then it has H-depth $2 n+1$ (and depth $2 n$ ). Define minimum depth $d(B, A)$, and minimum H-depth $d_{H}(B, A)$ to be the least depth, or H-depth, satisfied by $B \subseteq A$; if $B \subseteq A$ does not have finite depth, equivalently finite $\mathrm{H}$-depth, set $d(B, A)=d_{H}(B, A)=\infty$.

For example, $B \subseteq A$ has depth 1 iff ${ }_{B} A_{B}$ and ${ }_{B} B_{B}$ are H-equivalent 
[2]. Equivalently, ${ }_{B} A_{B} \oplus * \cong{ }_{B} B_{B}^{n}$ for some $n \in \mathbb{N}$ [21]. This in turn is equivalent to there being $f_{i} \in \operatorname{Hom}\left({ }_{B} A_{B},{ }_{B} B_{B}\right)$ and $r_{i} \in A^{B}$ such that $\operatorname{id}_{A}=\sum_{i} f_{i}(-) r_{i}$, the classical central projectivity condition [23]. In this case, it is easy to show that $A$ is ring isomorphic to $B \otimes_{Z(B)} A^{B}$ where $Z(B), A^{B}$ denote the center of $B$ and centralizer of $B$ in $A$. From this we deduce immediately that a centrally projective ring extension $A \supseteq B$ (equivalently, depth 1 extension) has centers satisfying $Z(B) \subseteq Z(A)$, a condition of Burciu that characterizes depth 1 for a semisimple complex subalgebra pair $B \subseteq A$. Depth 1 subgroups are normal with one other condition on centralizers that depends on the commutative ground ring [3].

For another and important example of depth, the subring $B \subset A$ has right depth 2 iff ${ }_{A} A_{B}$ and ${ }_{A} A \otimes_{B} A_{B}$ are similar; equivalently,

$$
{ }_{A} A \otimes_{B} A_{B} \oplus * \cong{ }_{A} A_{B}^{n}
$$

for some $n \in \mathbb{N}$. If $A=K G$ is a group algebra of a finite group $G$, over a commutative ring $K$, and $B=K H$ is the group algebra of a subgroup $H<G$, then $B \subseteq A$ has right depth 2 iff $H$ is a normal subgroup of $G$ iff $B \subseteq A$ has left depth 2 [4]; a similar statement is true for a Hopf subalgebra $R \subseteq H$ of finite index and over any field [4]. For this and further reasons mentioned in the first paragraphs of this section we propose the following terminology that is consistent with the literature on normality of subobjects [4], [2], [6] and of Galois extensions [16], [18].

Definition 1.7 Suppose that $B \subseteq A$ is a subring pair. We say that $B$ is a right (or left) normal subring of $A$ if $B \subseteq A$ satisfies the right (or left) depth 2 condition above. Similarly, if $B \rightarrow A$ is a ring homomorphism, we say that the ring extension $A \mid B$ is a right (or left) normal extension if the bimodules induced by $B \rightarrow A$ satisfy the right (or left) depth 2 condition. A normal extension or normal subring is both left and right normal.

For example, centrally projective, or depth 1 , ring extensions are normal extensions. As a corollary of [21, Theorem 3.2] we know that a QF extension is left normal if and only if it is right normal (extending the equivalence of left and right normality for Frobenius extensions in [16]). The Galois theory of a normal extension $A \supseteq B$ with the additional condition that $A_{B}$ is a balanced module (with respect to its endomorphism ring End $A_{B}$ ) is briefly summarized as follows: the $\operatorname{ring} T:=\left(A \otimes_{B} A\right)^{B} \cong \operatorname{End}_{A} A \otimes_{B} A_{A}$ has right 
bialgebroid structure (and is left projective) over the centralizer subring $A^{B}:=R[16]$ with coaction on $A$, denoted by $a \mapsto a_{(0)} \otimes_{R} a_{(1)} \in A \otimes_{R} T$, having coinvariant subring $B$, such that

$$
A \otimes_{B} A \stackrel{\cong}{\longrightarrow} A \otimes_{R} T
$$

given by the canonical Galois mapping $a \otimes_{B} c \mapsto a c_{(0)} \otimes_{R} c_{(1)}$ with inverse given by $a \otimes_{R} t \mapsto a t^{1} \otimes_{B} t^{2}[18]$.

\subsection{Sugano's theorem}

Compiling results in [25] into a theorem and using the terminology of depth, we provide a different proof (except in (7) below). Let $A \supseteq B \supseteq C$ be a tower of rings, and consider the centralizers $D:=A^{C} \supseteq A^{B}:=R$.

Theorem 1.8 Suppose $B$ is a left relative $H$-separable extension of $C$ in $A$; i.e., ${ }_{B} B \otimes_{C} A_{A} \oplus * \cong{ }_{B} A_{A}^{n}$. Then the following hold:

(1) $D$ is a left finitely generated projective module over its subring $R$;

(2) as natural $B$-A-bimodules, $B \otimes_{C} A \cong \operatorname{Hom}\left({ }_{R} D,{ }_{R} A\right)$ via $b \otimes a \longmapsto(d \mapsto$ $b d a)$;

(3) if $B$ is a split extension of $C$, then $D$ is a separable extension of $R$;

(4) if $d(C, B)=1$, then $d_{H}(R, D)=1$;

(5) if $B \supseteq C$ is a separable extension, then $D \supseteq R$ is a split extension;

(6) if $d_{H}(C, B)=1$, then $d(R, D)=1$;

(7) if $A^{R}=B$ (i.e. $B$ has the double centralizer property in $A$ ) and $B_{C}$ is a finitely generated projective module, then the isomorphism given in (2) restricts to $B \otimes_{C} B \cong \operatorname{Hom}\left({ }_{R} D_{R},{ }_{R} A_{R}\right)$ and (a) $d_{H}(C, B)=1$ iff $d(R, D)=1$; (b) $B \supseteq C$ is separable iff $D \supseteq R$ is split.

Proof. The relative H-separability condition (1) on the tower $A \supseteq B \supseteq C$ is clearly equivalent to the two conditions

- there are $g_{1}, \ldots, g_{n} \in \operatorname{Hom}\left({ }_{B} B \otimes_{C} A_{A},{ }_{B} A_{A}\right) \cong D$ via $g_{i} \mapsto g_{i}\left(1 \otimes_{C} 1\right)$, and $f_{1}, \ldots, f_{n} \in \operatorname{Hom}\left({ }_{B} A_{A},{ }_{B} B \otimes_{C} A_{A}\right) \cong\left(B \otimes_{C} A\right)^{B}$ via $f_{i} \mapsto f_{i}(1)$ such that $\sum_{i=1}^{n} f_{i} \circ g_{i}=\operatorname{id}_{B \otimes_{C} A}$;

- there are $e_{i} \in\left(B \otimes_{C} A\right)^{B}$ and $d_{i} \in D$ for $i=1, \ldots, n$ such that $1 \otimes_{C} 1=\sum_{i=1}^{n} e_{i} d_{i}$,

since we define $e_{i}=f_{i}(1)$ and $d_{i}=g_{i}\left(1 \otimes_{C} 1\right) \in A^{C}$. We will make use of the equation 


$$
1 \otimes_{C} 1=\sum_{i} e_{i}^{1} \otimes e_{i}^{2} d_{i}
$$

in almost every step of the proof below.

(1) Given $d \in D, d=\sum_{i} e_{i}^{1} d e_{i}^{2} d_{i}$. Define $h_{i} \in \operatorname{Hom}\left({ }_{R} D,{ }_{R} R\right)$ by $h_{i}(d)=$ $e_{i}^{1} d e_{i}^{2}$, thus $d=\sum_{i} h_{i}(d) d_{i}$ is a finite projective bases equation.

(2) An inverse to $b \otimes_{C} a \mapsto \lambda_{b} \circ \rho_{a}$ is given by sending $f \in \operatorname{Hom}\left({ }_{R} D,{ }_{R} A\right)$ into $\sum_{i} e_{i} f\left(d_{i}\right)$.

(3) Given a bimodule projection $E: B \rightarrow C$, note that applying $E$ to Eq. (4) yields $1=\sum_{i} E\left(e_{i}^{1}\right) e_{i}^{2} d_{i}$. At the same time, a computation shows that $\sum_{i} E\left(e_{i}^{1}\right) e_{i}^{2} \otimes_{R} d_{i} \in\left(D \otimes_{R} D\right)^{D}$.

(4) Trivially $D \cong \operatorname{Hom}\left({ }_{C} C_{C},{ }_{C} A_{C}\right)$, while $D \otimes_{R} D \cong \operatorname{Hom}\left({ }_{C} B_{C},{ }_{C} A_{C}\right)$ via $d \otimes_{R} d^{\prime} \mapsto \lambda_{d} \circ \rho_{d^{\prime}}$ (with inverse given by $g \mapsto \sum_{i} g\left(e_{i}^{1}\right) e_{i}^{2} \otimes_{R} d_{i}$ for each $\left.g \in \operatorname{Hom}\left({ }_{C} B_{C},{ }_{C} A_{C}\right)\right)$. The mapping $\mu: D \otimes_{R} D \rightarrow D$ corresponds under these isomorphisms to restriction $r: \operatorname{Hom}\left({ }_{C} B_{C},{ }_{C} A_{C}\right) \rightarrow$ $\operatorname{Hom}\left({ }_{C} C_{C},{ }_{C} A_{C}\right)$. If we have the depth one condition ${ }_{C} B_{C} \oplus * \cong{ }_{C} C_{C}^{m}$, then after applying the additive functor $\operatorname{Hom}\left(-,{ }_{C} A_{C}\right)$ and the $(D$ bimodule) isomorphisms just considered, we obtain $D \otimes_{R} D \oplus * \cong D^{m}$ as $D$ - $D$-bimodules, the $\mathrm{H}$-depth one condition.

(5) If $e \in\left(B \otimes_{C} B\right)^{B}$ satisfies $e^{1} e^{2}=1$, then the mapping in (2) applied to $e$ is a bimodule projection in $\operatorname{Hom}\left({ }_{R} D_{R},{ }_{R} R_{R}\right)$.

(6) If $B$ is an H-separable extension of $C$, there are $t$ elements $z_{i} \in\left(B \otimes_{C}\right.$ $B)^{B}$ and $t$ elements $r_{i} \in B^{C}$ such that $1 \otimes_{C} 1=\sum_{i=1}^{t} z_{i} r_{i}$ [14]. But $B^{C} \subseteq A^{C}=D$ and $d \mapsto z_{i}^{1} d z_{i}^{2}$ defines $t$ mappings $h_{i} \in \operatorname{Hom}\left({ }_{R} D_{R},{ }_{R} R_{R}\right)$ such that $d=\sum_{i} h_{i}(d) r_{i}$, a centrally projective bases equation for $D \supseteq$ $R$, thus $d(R, D)=1$.

(7) First note that ${ }_{A} A \otimes_{R} D_{D} \cong{ }_{A} \operatorname{Hom}\left(B_{C}, A_{C}\right)_{D}$ via $a \otimes d \mapsto \lambda_{a} \circ \rho_{d}$ (with inverse $\left.f \mapsto \sum_{i} f\left(e_{i}^{1}\right) e_{i}^{2} \otimes_{R} d_{i}\right)$. The isomorphism in (2) restricts to the composite isomorphism (using Proposition 20.11 in [1]) of

$$
\begin{aligned}
{ }_{B} B \otimes_{C} B_{B} & \cong{ }_{B} B \otimes_{C} \operatorname{Hom}\left({ }_{A} A_{R},{ }_{A} A_{R}\right)_{B} \\
& \cong{ }_{B} \operatorname{Hom}\left({ }_{A} \operatorname{Hom}\left(B_{C}, A_{C}\right)_{R},{ }_{A} A_{R}\right)
\end{aligned}
$$

(since $A^{R}=B\left(\cong \operatorname{Hom}\left({ }_{A} A,{ }_{A} A\right)^{R}\right)$ and $B_{C}$ is finite projective)

$$
\begin{aligned}
\cong{ }_{B} \operatorname{Hom}\left({ }_{A} A \otimes_{R} D_{R},{ }_{A} A_{R}\right)_{B} & \cong \operatorname{Hom}\left({ }_{R} D_{R},{ }_{R} \operatorname{Hom}\left({ }_{A} A,{ }_{A} A\right)_{R}\right) \\
& \cong{ }_{B} \operatorname{Hom}\left({ }_{R} D_{R},{ }_{R} A_{R}\right)_{B} .
\end{aligned}
$$


(8) $(7 \mathrm{a} \Leftarrow)$ Suppose $h_{j} \in \operatorname{Hom}\left({ }_{R} D_{R},{ }_{R} R_{R}\right)$ and $w_{j} \in D^{R}$ satisfy $\operatorname{id}_{D}=$ $\sum_{j} h_{j}(-) w_{j}$. Then using the isomorphism in (7) there are $e_{j} \in\left(B \otimes_{C}\right.$ $B)^{B} \cong \operatorname{Hom}\left({ }_{R} D_{R},{ }_{R} R_{R}\right)$ such that $h_{j}(d)=e_{j}^{1} d e_{j}^{2}$ for all $d \in D$. Note that $w_{j} \in D^{R} \subseteq A^{R}=B$ and $w_{j} \in D=A^{C}$, whence $w_{j} \in B^{C}$. It follows from the isomorphism (7) that $1 \otimes_{C} 1=\sum_{j} e_{j} w_{j}$ an equivalent condition for H-separability, $d_{H}(C, B)=1$.

(9) $(7 \mathrm{~b} \Leftarrow)$ Given a projection $E:{ }_{R} D_{R} \rightarrow{ }_{R} R_{R}$ one notes that $E \in$ $\operatorname{Hom}\left({ }_{R} D_{R},{ }_{R} A_{R}\right)^{B} \cong\left(B \otimes_{C} B\right)^{B}$, so that there is $e \in\left(B \otimes_{C} B\right)^{B}$ such that $E(d)=e^{1} d e^{2}$ for all $d \in D$. In particular, $e^{1} e^{2}=E(1)=1$.

Since H-separability implies separability for ring extensions, we might expect some mild condition should imply the same for towers of rings. The next corollary addresses this question.

Corollary 1.9 Suppose $A \supseteq B \supseteq C$ is a left relative $H$-separable tower satisfying $D=A^{C}$ is a left split extension of $R=A^{B}$. Then $A \supseteq B \supseteq C$ is left relative separable.

Proof. Applying (2) of Sugano's theorem, note that $\mu: B \otimes_{C} A \rightarrow A$ corresponds to the $A$-dual of the inclusion $\iota:{ }_{R} R \rightarrow{ }_{R} A$, which is $\iota^{*}$ : $\operatorname{Hom}\left({ }_{R} D,{ }_{R} A\right) \rightarrow \operatorname{Hom}\left({ }_{R} R,{ }_{R} A\right) \cong{ }_{B} A_{A}$. If $\iota$ is a split monic, then $\iota^{*}$ and $\mu$ are split $B$ - $A$-epimorphisms.

Corollary 1.10 Suppose $K$ is a commutative ring, $A$ is a $K$-algebra with $B$ a $K$-subalgebra satisfying the $B$-A-bimodule generator condition $B \otimes_{K}$ $A \oplus * \cong A^{n}$ (for some $\left.n \in \mathbb{N}\right)$. Let $R$ be the centralizer $A^{B}$. The following holds:

(1) ${ }_{R} A$ is a finite projective module;

(2) $B \otimes_{K} A \cong$ End $_{R} A$ via $b \otimes a \mapsto \lambda_{b} \circ \rho_{a}$;

(3) if $B$ has a $K$-linear projection onto $K 1$, then $A$ is a separable extension of $R$;

(4) if $B$ is finite projective as a $K$-module, then $A$ is an $H$-separable extension of $R$;

(5) if $B$ is a separable $K$-algebra, then $A$ is a progenerator $B$-A-bimodule and $A \supseteq R$ is a split extension;

(6) if $B$ is an Azumaya algebra with center $Z$ such that $Z \otimes_{K} Z \cong Z$ (via $\mu)$, then $A$ is centrally projective over its subalgebra $R$;

(7) if $A^{R}=B$ and $B$ is a finite projective $K$-module, then the isomorphism 
in (2) restricts to $B \otimes_{K} B \cong \operatorname{End}_{R} A_{R}$ and (5), (6) become iff statements.

Proof. The proof follows from Sugano's theorem by letting $C=K 1$, the unit subalgebra in $A$ and $B$. In (6) and (7) we make use of Sugano's characterization of an $\mathrm{H}$-separable $K$-algebra $B$ as being Azumaya over its center $Z$ subject to the condition $\mu: Z \otimes_{K} Z \stackrel{\cong}{\longrightarrow} Z$. In (5), the bimodule ${ }_{B} A_{A}$ is already noted to be a generator, and it is finite projective, since given any $B$ - $A$-bimodule ${ }_{B} M_{A}$ and $B$ - $A$-epimorphism $\phi: M \rightarrow A, \phi$ is split by $a \mapsto e^{1} m e^{2} a$ where $e \in B^{e}$ is a separability idempotent and $\phi(m)=1$.

A converse to Lemma 1.5 is given in the following. The hypothesis of cleft extension in the corollary is fullfilled for example by any finitedimensional $A$ with nilradical $J$ and separable subalgebra $B \cong A / J$ (using Wedderburn's Principal Theorem).

Corollary 1.11 Suppose $\pi:{ }_{B} A_{B} \rightarrow{ }_{B} B_{B}$ is a ring epimorphism splitting $A \supseteq B$ (a so-called cleft extension), and $C$ is a subring of $B$ such that the left relative $H$-separable tower condition holds. Then $B \supseteq C$ is $H$-separable (i.e., $\left.d_{H}(C, B)=1\right)$.

Proof. Apply id $\operatorname{id}_{B} \otimes_{C} \pi$ to the decomposition of $1 \otimes_{C} 1$ given in Eq. (4). We obtain $1 \otimes_{C} 1=\sum_{i} e_{i}^{1} \otimes \pi\left(e_{i}^{2}\right) \pi\left(d_{i}\right)$ where each $e_{i}^{1} \otimes_{C} \pi\left(e_{i}^{2}\right) \in\left(B \otimes_{C} B\right)^{B}$ and each $\pi\left(d_{i}\right) \in B^{C}$ : possessing Casimir elements and centralizer elements like these characterizes H-separability of $B$ over $C$.

\section{Subring depth in a relative separable tower}

The progenerator condition in Corollary 1.10 is used again in the hypothesis of the proposition below.

Proposition 2.1 Suppose a finitely generated projective $K$-algebra $A$ has subalgebra $B$ such that $A$ is a progenerator $B$-A-bimodule. Then $A \supseteq B$ is left normal.

Proof. Since $\mu: B \otimes_{K} A \rightarrow A$ splits, ${ }_{B} A_{A} \oplus * \cong B \otimes_{K} A$; thus tensoring by $A \otimes_{B}$ - we obtain $A \otimes_{B} A \oplus * \cong A \otimes_{K} A$ as natural $A$-bimodules. Since $B$ is a separable algebra, any $B$-module is $K$-relative projective, whence by the hypothesis on $A,{ }_{B} A \oplus * \cong{ }_{B} B^{m}$ and so $A \otimes_{B} A \oplus * \cong B \otimes_{K} A^{m}$ as $B$ $A$-bimodules. Since ${ }_{B} A_{A}$ is a generator, it follows that $B \otimes_{K} A \oplus * \cong{ }_{B} A_{A}^{q}$, whence ${ }_{B} A \otimes_{B} A_{A} \oplus * \cong{ }_{B} A_{A}^{m q}$, the left depth 2 condition on $A \supseteq B$. 
Proposition 2.2 Suppose a tower of rings $A \supseteq B \supseteq C$ satisfies the left relative $H$-separability condition ${ }_{B} B \otimes_{C} A_{A} \oplus * \cong{ }_{B} A_{A}^{n}$ and the left relative separability condition ${ }_{B} A_{A} \oplus * \cong_{B} B \otimes_{C} A_{A}$. Then $d_{H}(B, A)=d_{H}(C, A)$.

Proof. Tensoring by ${ }_{A} A \otimes_{B}$ - the left relative H-separability condition yields ${ }_{A} A \otimes_{C} A_{A} \oplus * \cong{ }_{A} A \otimes_{B} A_{A}^{n}$. Tensoring by ${ }_{A} A \otimes_{B}$ - the left relative separability condition above yields $A \otimes_{B} A \oplus * \cong A \otimes_{C} A$ as natural $A$ bimodules, whence $A \otimes_{B} A$ and $A \otimes_{C} A$ are H-equivalent as $A$ - $A$-bimodules.

Suppose that $A^{\otimes_{B} n}$ is H-equivalent to $A^{\otimes_{C} n}$ for any $m>n \geq 2$. Then $A^{\otimes_{B}(m-1)}$ and $A^{\otimes_{C}(m-1)}$ are H-equivalent, so $A \otimes_{C} A^{\otimes_{B}(m-1)}$ and $A^{\otimes_{C} m}$ are H-equivalent, as are $\left(A \otimes_{C} A\right) \otimes_{B} \cdots \otimes_{B} A$ and $\left(A \otimes_{B} A\right) \otimes_{B} \cdots \otimes_{B} A$. It follows from this inductive argument that $A^{\otimes_{B} m}$ and $A^{\otimes_{C} m}$ are H-equivalent as $A$-bimodules for any $m>1$.

Suppose $A \supseteq B$ has H-depth 1, equivalently, $A$ and $A \otimes_{B} A$ are $\mathrm{H}-$ equivalent, which is equivalent to $A$ and $A \otimes_{C} A$ being H-equivalent iff $A \supseteq C$ has H-depth 1. From the definition of H-depth in Section 1 and the H-equivalences noted above, $A \supseteq B$ has H-depth $n$ iff $A \supseteq C$ has H-depth $n$ for any $n \geq 1$.

We improve on [17, Theorem 2.3] next.

Proposition 2.3 Suppose $B$ is an Azumaya $K$-algebra and subalgebra of a finitely generated projective $K$-algebra $A$. Then $A \supseteq B$ has depth 1 .

Proof. Since $B$ is Azumaya, it is well-known that $B$ is a progenerator $B^{e_{-}}$ module (e.g. [14]). Since $B$ is a separable $K$-algebra, $B^{e}$ is a semisimple extension of $K 1$. Then ${ }_{B} A_{B}$ is $K$-relative projective, therefore ${ }_{B} A_{B}$ is finite projective since $A$ is projective over $K$. Thus $A \oplus * \cong B \otimes_{K} B^{m}$ for some $m \in \mathbb{N}$. But $B^{e} \oplus * \cong{ }_{B} B_{B}^{n}$ for some $n \in \mathbb{N}$ since ${ }_{B} B_{B}$ is a generator. Putting these together, ${ }_{B} A_{B} \oplus * \cong{ }_{B} B_{B}^{m n}$.

\subsection{Higman-Jans-like theorem}

Higman's theorem in [10] states that a finite-dimensional group algebra $k G$, where $k$ is a field of positive characteristic $p$, has finite representation type if and only if the Sylow $p$-subgroup of $G$ is cyclic. The proof was teased apart by Jans in [13] into two statements about the property of finite representation type of a subalgebra pair of Artin algebras going up or down according to whether $A$ is a split or separable extension of $B$; e.g., a separable and finitely generated extension $A \supseteq B$ of Artin algebras where $B$ 
has finitely many isoclasses of indecomposable modules implies that also $A$ has finite representation type; see also [24, pp. 173-174]. In generalizing this theorem, we first need a lemma characterizing left relative separable towers of rings $A \supseteq B \supseteq C$ in terms of modules.

Lemma 2.4 $B$ is a left relative separable extension of $C$ in $A$ if and only if for each module ${ }_{A} M$, the mapping $\mu_{M}: B \otimes_{C} M \rightarrow M$ given by $b \otimes_{C} m \mapsto b m$ splits naturally as a left B-module epimorphism.

Proof. $\quad(\Rightarrow)$ This is clear from tensoring the split epi $\mu: B \otimes_{C} A \rightarrow A$ by $-\otimes_{A} M$ to obtain the split epi $\mu_{M} . \quad(\Leftarrow)$ Apply the hypothesis to $M=A$ and use naturality to obtain a split $B$ - $A$-bimodule epi $\mu: B \otimes_{C} A \rightarrow A$.

Let $A$ be an Artin algebra, $A-\bmod$ denote the category of finitely generated left $A$-modules, and add $M$ denote the category of summands of finite sums of copies of a module $M$.

Theorem 2.5 Suppose $A \supseteq B \supseteq C$ is a left relative separable tower of Artin algebras, where ${ }_{B} A$ and ${ }_{C} B$ are finitely generated. Suppose $C-\bmod$ has finitely many isoclasses of indecomposable representatives $V_{1}, \ldots, V_{n}$. Then the restriction functor $\operatorname{Res}_{B}^{A}: A-\bmod \rightarrow B-\bmod$ factors through the subcategory add $\bigoplus_{i=1}^{n} B \otimes_{C} V_{i}$.

Proof. Given $M \in A-\bmod$, its restrictions ${ }_{B} M$ and ${ }_{C} M$ are finitely generated. By the lemma, ${ }_{B} M$ is isomorphic to a direct summand of $B \otimes_{C} M$. Since the restriction ${ }_{C} M \cong \bigoplus_{i=1}^{n} n_{i} V_{i}$ for some nonnegative integers $n_{i}$, one obtains $\operatorname{Res}_{B}^{A} M \oplus * \cong \bigoplus_{i=1}^{n} n_{i} B \otimes_{C} V_{i}$, which is expressible as a KrullSchmidt decomposition into finitely many indecomposable $B$-module summands of $B \otimes_{C} V_{1}, \ldots, B \otimes_{C} V_{n}$.

Of course if $A=B$ and $B \supseteq C$ is a separable finitely generated extension, then the theorem recovers Jans's, " $B$ has finite representation type if $C$ has."

\subsection{Triviality of Relatively H-separable Group Algebra Towers}

We next note that towers of finite complex group algebras that are left or right relative H-separable extensions are just arbitrary group algebra extensions.

Proposition 2.6 Let $A=\mathbb{C} G \supseteq B=\mathbb{C} H \supseteq C=\mathbb{C} J$ where $G>H>J$ is a tower of subgroups of a finite group $G$. Then $A \supseteq B \supseteq C$ is a left or 
right relative $H$-separable tower of algebras if and only if $H=J$.

Proof. Given ${ }_{B} B \otimes_{C} A_{A} \oplus * \cong{ }_{B} A_{A}^{n}$, we tensor this with simple left $A$ modules, i.e. $G$-modules, and make use of their (irreducible) characters. Let $\psi \in \operatorname{Irr}(G)$ and $\phi \in \operatorname{Irr}(H)$. From the relative H-separable condition above it follows that

$$
\left\langle\operatorname{Ind}_{J}^{H} \operatorname{Res}_{J}^{G} \psi, \phi\right\rangle_{H} \leq n\left\langle\operatorname{Res}_{H}^{G} \psi, \phi\right\rangle_{H}
$$

Letting $\psi=1_{G}$, note that $\operatorname{Res}_{J}^{G} 1_{G}=1_{J}$ for instance, so that

$$
\left\langle\operatorname{Ind}_{J}^{H} 1_{J}, \phi\right\rangle_{H} \leq n\left\langle 1_{H}, \phi\right\rangle_{H}
$$

This last inner product is zero if $\phi \neq 1_{H}$, so that also $\left\langle\operatorname{Ind}_{J}^{H} 1_{J}, \phi\right\rangle_{H}=$ 0 . If $\phi=1_{H}$, then $\left\langle\operatorname{Ind}_{J}^{H} 1_{J}, 1_{H}\right\rangle_{H}=\left\langle 1_{J}, \operatorname{Res}_{J}^{H} 1_{H}\right\rangle_{J}=1$ by Frobenius reciprocity. From the orthonormal expansion of $\operatorname{Ind}_{J}^{H} 1_{J}$ in terms of $\operatorname{Irr}(H)$, it follows that $\operatorname{Ind}_{J}^{H} 1_{J}=1_{H}$. Comparing degrees, it follows that $|H: J|=1$, whence $H=J$. The proof using the right relative $\mathrm{H}$-separability condition is a similar use of characters of right modules. The converse is of course trivial.

We have seen in Lemma 1.2 that a tower $A \supseteq B \supseteq C$ of arbitrary finite group algebras is always left or right relative separable if $B \supseteq C$ is a separable extension (iff $|H: J|$ is invertible in the ground ring). This follows from the fact that group algebra extensions are split extensions (since given a subgroup $H<G$, the difference set $G-H$ is closed under multiplication by $H)$.

\section{A Characterization of normality for progenerator ring exten- sions}

The next proposition provides an alternative characterization of the left relative H-separable condition for a tower $A \supseteq B \supseteq C$ where $B_{C}$ is finitely generated and projective.

Proposition 3.1 Suppose $A \supseteq B \supseteq C$ is a tower of rings such that the natural module $B_{C}$ is finite projective. Then the left relative $H$-separable condition (1) is equivalent to the condition, $(\exists n \in \mathbb{N}:)$

$$
{ }_{A} \operatorname{Hom}\left(B_{C}, A_{C}\right)_{B} \oplus * \cong{ }_{A} A_{B}^{n} .
$$


Proof. Apply $\operatorname{Hom}\left(-{ }_{A}, A_{A}\right)$, an additive functor from the category of $B-A$ bimodules into the category of $A$ - $B$-bimodules, to (1), ${ }_{B} B \otimes_{C} A_{A} \oplus * \cong{ }_{B} A_{A}^{n}$. Note that $\operatorname{Hom}\left(B \otimes_{C} A_{A}, A_{A}\right) \cong \operatorname{Hom}\left(B_{C}, A_{C}\right)$ as natural $A$ - $B$-bimodules via $F \mapsto F\left(-\otimes_{C} 1\right)$ with inverse

$$
f \longmapsto\left(b \otimes_{C} a \mapsto f(b) a\right)
$$

for every $f \in \operatorname{Hom}\left(B_{C}, A_{C}\right)$. Since ${ }_{A} \operatorname{Hom}\left(A_{A}, A_{A}\right)_{B} \cong{ }_{A} A_{B}$, the condition (5) follows without the assumption that $B_{C}$ is finite projective.

Assuming that $B_{C}$ is finite projective, it follows that $B \otimes_{C} A_{A}$ is finite projective and therefore reflexive. Then $\operatorname{Hom}\left({ }_{A} \operatorname{Hom}\left(B_{C}, A_{C}\right),{ }_{A} A\right) \cong B \otimes_{C}$ $A$ as natural $B$ - $A$-bimodules. It follows reflexively that condition (5) implies condition (1).

The next theorem provides many interesting classes of examples of relative $\mathrm{H}$-separable towers of rings.

Theorem 3.2 Suppose $B \supseteq C$ is a ring extension and has the natural module $B_{C}$ a progenerator. Let $A:=\operatorname{End} B_{C}$ and $B \hookrightarrow A$ given by the left regular representation $b \mapsto \lambda_{b}$. Then $B \supseteq C$ is right normal if and only if the tower $A \supseteq B \supseteq C$ is left relative $H$-separable.

Proof. $\quad(\Rightarrow)$ This direction of the proof only requires that $B_{C}$ is finite projective. Given the right normality condition,

$$
{ }_{B} B \otimes_{C} B_{C} \oplus * \cong{ }_{B} B_{C}^{m}
$$

for some $m \in \mathbb{N}$, apply the bimodule ${ }_{A} B_{C}$ and the additive functor ${ }_{A} \operatorname{Hom}\left(-{ }_{C}, B_{C}\right)$ to this. Note that the hom-tensor adjoint relation implies that $\operatorname{Hom}\left(B \otimes_{C} B_{C}, B_{C}\right) \cong \operatorname{Hom}\left(B_{C}, \operatorname{Hom}\left(B_{C}, B_{C}\right)_{C}\right)$ as natural $A$ $B$-bimodules. This implies condition (5), equivalent by the proposition to (1).

$(\Leftarrow)$ Since we assume $B_{C}$ is a progenerator, the rings $C$ and $A$ are Morita equivalent, with bimodules ${ }_{A} B_{C}$ and ${ }_{C} \operatorname{Hom}\left(B_{C}, C_{C}\right)_{A}$ forming a Morita context. In particular, $\operatorname{Hom}\left(B_{C}, C_{C}\right) \otimes_{A} B_{C} \cong C$ as $C$-bimodules and $B \otimes_{C} \operatorname{Hom}\left(B_{C}, C_{C}\right) \cong A$ as $A$-bimodules.

Supposing that condition (5) holds on the tower $C \subseteq B \hookrightarrow A$, we substitute ${ }_{A} A_{B}={ }_{A} \operatorname{Hom}\left(B_{C}, B_{C}\right)_{B}$ in this condition and apply the homtensor adjoint relation with the last Morita isomorphism to obtain: 


$$
\operatorname{Hom}\left(B \otimes_{C} B_{C},{ }_{A} B_{C}\right)_{B} \oplus * \cong{ }_{A} B \otimes_{C} \operatorname{Hom}\left(B_{C}, C_{C}\right)_{B}^{n}
$$

Tensor this from the left by the additive functor ${ }_{C} \operatorname{Hom}\left(B_{C}, C_{C}\right) \otimes_{A}-$ and using the (cancellation) isomorphism of Morita pointed out above. We obtain ${ }_{C} \operatorname{Hom}\left(B \otimes_{C} B_{C}, \operatorname{Hom}\left(B_{C}, C_{C}\right) \otimes_{A} B_{C}\right)_{B} \oplus * \cong$

$$
{ }_{C} \operatorname{Hom}\left(B \otimes_{C} B_{C}, C_{C}\right)_{B} \oplus * \cong{ }_{C} \operatorname{Hom}\left(B_{C}^{n}, C_{C}\right)_{B}
$$

since $B \otimes_{C} B_{C}$ is finite projective and one may apply the well-known natural isomorphism [1, Proposition 20.10]. Now by reflexivity of the projective modules $B_{C}^{n}$ and $B \otimes_{C} B_{C}$, we apply to this last isomorphism the additive functor $\operatorname{Hom}\left(-,{ }_{C} C\right)$ from the category of $C$-B-bimodules into the category of $B$-C-bimodules and obtain the condition (6) with $n=m$.

The left and right relative $\mathrm{H}$-separable conditions on a tower $A \supseteq$ $B \supseteq C$ are equivalent if $B \supseteq C$ is a Frobenius extension, i.e., ${ }_{C} B_{B} \cong$ ${ }_{C} \operatorname{Hom}\left(B_{C}, C_{C}\right)_{B}$ as bimodules and $B_{C}$ has finite projective bases $\left\{b_{i}\right\} \subset B$, $\left\{\phi_{i}\right\} \subset \operatorname{Hom}\left(B_{C}, C_{C}\right),(i=1, \ldots, m)$.

Proposition 3.3 If $B \supseteq C$ is a Frobenius extension, then a tower $A \supseteq B \supseteq C$ is left relative $H$-separable if and only if it is right relative H-separable.

Proof. We make use of the equivalent condition for left relative H-separable tower in Proposition 3.1. We note that $\operatorname{Hom}\left(B_{C}, A_{C}\right) \cong A \otimes_{C} \operatorname{Hom}\left(B_{C}, C_{C}\right)$ via $f \mapsto \sum_{i} f\left(b_{i}\right) \otimes_{C} \phi_{i}$, with inverse given by the "one-point projections" mapping $a \otimes_{C} \psi \mapsto a \psi(-)$. Observe that this mapping is an $A$ $B$-bimodule isomorphism. It follows from the Frobenius condition ${ }_{C} B_{B} \cong$ ${ }_{B} \operatorname{Hom}\left(B_{C}, C_{C}\right)_{B}$ that the right relative $\mathrm{H}$-separable condition is satisfied by $A \supseteq B \supseteq C$.

As a corollary of this proposition and Theorem 3.2, we note that the right normality condition for Frobenius extensions with surjective Frobenius homomorphism is equivalent to left normality condition, another proof in this case of [16].

Corollary 3.4 If $B \supseteq C$ is a Frobenius extension, where $B_{C}$ is a generator, then $B \supseteq C$ is left normal if and only if it is right normal. 


\subsection{A characterization of normality for Frobenius extensions}

In this subsection we characterize normal (twisted) Frobenius extensions together with their endomorphism rings as being relative $\mathrm{H}$-separable towers. We find it convenient to change notation to $A \supseteq B$ being the Frobenius ring extension and $E:=$ End $A_{B}$ being the top ring in the tower $B \subseteq A \hookrightarrow E$ where $A \hookrightarrow E$ is given by $a \mapsto \lambda_{a}$ and $\lambda_{a}(x)=a x$.

Suppose $\beta: B \rightarrow B$ is a ring automorphism of $B$. Denote a $B$-module $M_{B}$ as $M_{\beta}$ if twisted by $\beta$ as follows: $m \cdot b=m \beta(b)$. Recall that a $\beta$ Frobenius (ring) extension $A \supseteq B$ is characterized by having a (Frobenius) homomorphism $F:{ }_{B} A_{B} \rightarrow{ }_{\beta} B_{B}$ satisfying $F\left(b_{1} a b_{2}\right)=\beta\left(b_{1}\right) F(a) b_{2}$ for each $b_{1}, b_{2} \in B, a \in A$. Dual bases $\left\{x_{i}\right\},\left\{y_{i}\right\}$ in $A$ satisfy $\sum_{i=1}^{n} x_{i} F\left(y_{i} a\right)=a$ and $\sum_{i=1}^{n} \beta^{-1}\left(F\left(a x_{i}\right)\right) y_{i}=a$ for each $a \in A$. Equivalently, $A_{B}$ is finite projective and $A \cong{ }_{\beta} \operatorname{Hom}\left(A_{B}, B_{B}\right)$ as $B$ - $A$-bimodules: see [14] for more details and references.

For example, if $\beta$ is an inner automorphism, then $A \supseteq B$ is an (ordinary) Frobenius extension, such as a group algebra extension of a group $G$ and subgroup $H$ of finite index $n$. (Suppose $g_{1}, \ldots, g_{n}$ are the right coset representatives of $H$ in $G, K$ an arbitrary commutative ring, then the group algebra $A=K G$ is a Frobenius extension of the group subalgebra $B=K H$ with $F: A \rightarrow B$ the obvious projection defined by $F\left(\sum_{g \in G} a_{g} g\right)=\sum_{h \in H} a_{h} h$ and dual bases $x_{i}=g_{i}^{-1}, y_{i}=g_{i}$.)

Corollary 3.5 Suppose $A \supseteq B$ is a $\beta$-Frobenius extension with surjective Frobenius homomorphism $F: A \rightarrow B$. Let $E:=$ End $A_{B}$ and embed $A \hookrightarrow E$ via the left regular representation $\lambda_{a}(x)=a x$. Then the tower of rings $B \subseteq A \hookrightarrow E$ is left relative H-separable if and only if $B \subseteq A$ is right normal.

Proof. Since $F: A \rightarrow B$ is assumed surjective, it follows that $A_{B}$ (and ${ }_{B} A$ by using equivalently $\beta^{-1} \circ F$ ) is a generator. It also follows from the hypothesis of Frobenius extension that $A_{B}$ (and ${ }_{B} A$ ) are finite projective. Apply Theorem 3.2 to conclude that the left relative $\mathrm{H}$-separable tower condition on $B \subseteq A \hookrightarrow E$ is equivalent to the right normality condition on $B \subseteq A$.

Recall that a Hopf subalgebra $R$ is normal in a Hopf algebra $H$ if $R$ is stable under the left and right adjoint actions of $H$ on $R$. For group algebra extensions this specializes to the usual notion of normal subgroup. 
Corollary 3.6 A Hopf subalgebra $R$ of a finite-dimensional Hopf algebra $H$ is normal if and only if the tower of algebras $R \subseteq H \hookrightarrow$ End $H_{R}$ is left relative $H$-separable.

Proof. This follows from Corollary 3.5 and theorems that $H$ is a $\beta$ Frobenius extension of $R$ (Oberst-Schneider), and another that $H_{R}$ is free (Nichols-Zoeller). Also, as remarked in the introduction, the equivalence of the normality condition for a Hopf subalgebra $R \subseteq H$ with the depth 2 condition on the ring extension $R \subseteq H$ follows from [2].

\subsection{Galois correspondence proposal}

Again let $E$ denote End $A_{B}$. The condition on the tower $B \subseteq A \hookrightarrow E$ in the next corollary is called the rD3 condition in [19]. The depth three condition on $A \supseteq B$ is that ${ }_{B} A \otimes_{B} A_{B} \oplus * \cong{ }_{B} A_{B}^{m}$ for some $m \in \mathbb{N}$. Below we apply the same Frobenius coordinate system as above, but we may assume that the twist automorphism $\beta=\mathrm{id}_{B}$.

Corollary 3.7 Suppose $A \supseteq B$ is a Frobenius extension with surjective Frobenius homomorphism. Then $A \supseteq B$ has depth 3 if and only if $E E \otimes_{A}$ $E_{B} \oplus * \cong{ }_{E} E_{B}^{m}$ for some $m \in \mathbb{N}$.

Proof. The proof is similar to the proof of Theorem 3.2, but using the $E$ $A$-bimodule isomorphism $E \stackrel{\cong}{\longrightarrow} A \otimes_{B} A$ given by $f \mapsto \sum_{i} f\left(x_{i}\right) \otimes y_{i}$, with inverse mapping given by $a \otimes_{B} a^{\prime} \mapsto \lambda_{a} \circ F \circ \lambda_{a^{\prime}}$. The rest of the proof is left to the reader.

The two conditions of "depth three" and "depth two" on a tower go up and down as follows. The short proof is left to the reader as an exercise using Proposition 1.1.

Proposition 3.8 Suppose $A \supseteq B \supseteq C \supseteq D$ is a tower of unital subrings. If $A \supseteq B$ has depth 1 and $A \supseteq B \supseteq C$ is right relative $H$-separable, then $B \supseteq C \supseteq D$ satisfies the $r D 3$ condition, ${ }_{B} B \otimes_{C} B_{D} \oplus * \cong{ }_{B} B_{D}^{n}$ for some $n \in \mathbb{N}$. If $B \supseteq D$ has $H$-depth 1 and $B \supseteq C \supseteq D$ satisfies the $r D 3$ condition, then $A \supseteq B \supseteq C$ is right relative $H$-separable.

In [25] the left relative separable and H-separable conditions on towers of rings are used by Sugano for Galois correspondence in an $\mathrm{H}$-separable extension in terms of centralizers. A final thought is to ask if results for Galois correspondence of a normal extension in [19] (in terms of endomorphism 
rings) may be improved with the use of the tower condition studied in this paper.

Acknowledgements Research for this paper was funded by the European Regional Development Fund through the programme COMPETE and by the Portuguese Government through the FCT under the project PE-C/MAT/UI0144/2013.nts.

\section{References}

[ 1 ] Anderson F. W. and Fuller K., Rings and Categories of Modules, G.T.M. 13, Springer, 1992.

[ 2 ] Boltje R. and Külshammer B., On the depth 2 condition for group algebra and Hopf algebra extensions. J. Algebra 323 (2010), 1783-1796.

[ 3 ] Boltje R. and Külshammer B., Group algebra extensions of depth one. Algebra Number Theory 5 (2011), 63-73.

[4] Boltje R., Danz S. and Külshammer B., On the depth of subgroups and group algebra extensions. J. Algebra 335 (2011), 258-281.

[ 5 ] Burciu S. and Kadison L., Subgroups of depth three. Surv. Diff. Geom. XV (2011), 17-36.

[ 6 ] Burciu S., Kadison L. and Külshammer B., On subgroup depth. I.E.J.A. 9 (2011), 133-166.

[ 7 ] Danz S., The depth of some twisted group algebra extensions. Comm. Alg. 39 (2011), 1-15.

[ 8 ] Fritzsche T., The depth of subgroups of PSL(2,q). J. Algebra 349 (2011), 217-233.

[ 9 ] Fritzsche T., Külshammer B. and Reiche C., The depth of Young subgroups of symmetric groups. J. Algebra 381 (2013), 96-109.

[10] Higman D. G., Indecomposable representations at characteristic p. Duke Math. J. 7 (1954), 377-381.

[11] Hirata K., Some types of separable extensions of rings. Nagoya Math. J. 33 (1968), 107-115.

[12] Hirata K. and Sugano K., On semisimple and separable extensions of noncommutative rings. J. Math. Soc. Japan 18 (1966), 360-373.

[13] Jans J.-P., The representation type of algebras and subalgebras. Can. J. Math. 10 (1957), 39-44.

[14] Kadison L., New examples of Frobenius extensions, University Lecture Series, 14, Amer. Math. Soc., Providence, 1999.

[15] Kadison L. and Nikshych D., Hopf algebra actions on strongly separable 
extensions of depth two. Adv. Math. 163 (2001), 312-342.

[16] Kadison L. and Szlachányi K., Bialgebroid actions on depth two extensions and duality. Adv. Math. 179 (2003), 75-121.

[17] Kadison L., Anchor maps and stable modules in depth two. Appl. Cat. Struct. 16 (2008), 141-157.

[18] Kadison L., Infinite index subalgebras of depth two. Proc. A.M.S. 136 (2008), 1523-1532.

[19] Kadison L., Finite depth and Jacobson-Bourbaki correspondence. J. Pure \& Appl. Alg. 212 (2008), 1822-1839.

[20] Kadison L., Odd H-depth and H-separable extensions. Cen. Eur. J. Math. 10 (2012), 958-968.

[21] Kadison L., Subring depth, Frobenius extensions and towers. Int. J. Math. \& Math. Sci., 2012, article 254791.

[22] Kadison L., Hopf subalgebras and tensor powers of generalized permutation modules. J. Pure \& Appl. Alg. 218 (2014), 367-380.

[23] Müller B., Quasi-Frobenius-Erweiterungen. Math. Z. 85 (1964), 345-368.

[24] Pierce R., Associative Algebras, G.T.M. 88, Springer, 1982.

[25] Sugano K., On centralizers in separable extensions. Osaka J. Math. 7 (1970), 29-40.

Departamento de Matematica

Faculdade de Ciências da Universidade do Porto

Rua Campo Alegre 687

4169-007 Porto

E-mail: lkadison@fc.up.pt 